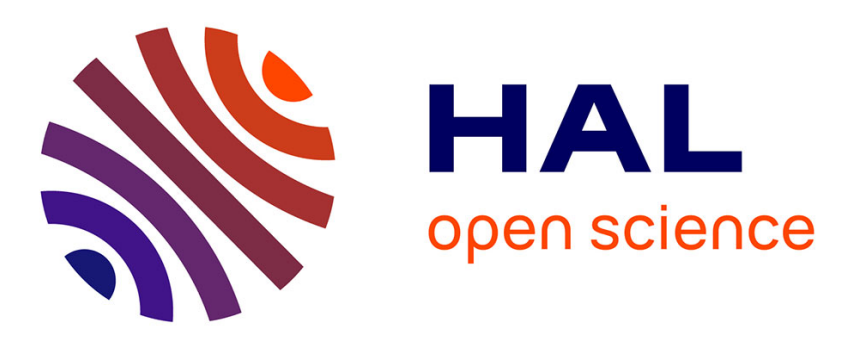

\title{
Dielectrophoretic Rayleigh-Bénard convection under microgravity conditions
}

Harunori Yoshikawa, Mireille Tadie Fogaing, Olivier Crumeyrolle, Innocent Mutabazi

\section{- To cite this version:}

Harunori Yoshikawa, Mireille Tadie Fogaing, Olivier Crumeyrolle, Innocent Mutabazi. Dielectrophoretic Rayleigh-Bénard convection under microgravity conditions. Physical Review E: Statistical, Nonlinear, and Soft Matter Physics, 2013, 87, pp.043003. 10.1103/PhysRevE.87.043003 . hal-00906494

\section{HAL Id: hal-00906494 \\ https://hal.science/hal-00906494}

Submitted on 19 Nov 2013

HAL is a multi-disciplinary open access archive for the deposit and dissemination of scientific research documents, whether they are published or not. The documents may come from teaching and research institutions in France or abroad, or from public or private research centers.
L'archive ouverte pluridisciplinaire HAL, est destinée au dépôt et à la diffusion de documents scientifiques de niveau recherche, publiés ou non, émanant des établissements d'enseignement et de recherche français ou étrangers, des laboratoires publics ou privés. 


\title{
Dielectrophoretic Rayleigh-Bénard convection under microgravity conditions
}

\author{
H. N. Yoshikawa, ${ }^{*}$ M. Tadie Fogaing, O. Crumeyrolle, and I. Mutabazi \\ Laboratoire Ondes et Milieux Complexes, UMR 6294 CNRS - Université du Havre \\ 53, rue de Prony, CS80540 - 76058 Le Havre Cedex, France
}

(Dated: March 14, 2013)

\begin{abstract}
Thermal convection in a dielectric fluid layer between two parallel plates subjected to an alternating electric field and a temperature gradient is investigated under microgravity conditions. A thermo-electric coupling resulting from the thermal variation of the electric permittivity of the fluid produces the dielectrophoretic (DEP) body force, which can be regarded as thermal buoyancy due to an effective gravity. This electric gravity can destabilize a stationary conductive state of the fluid to develop convection. The similarity of the DEP thermal convection with the Rayleigh-Bénard (RB) convection is examined by considering its behavior in detail by a linear stability theory and a two-dimensional direct numerical simulation. The results are analyzed from an energetic viewpoint and in the framework of the Ginzburg-Landau (GL) equation. The stabilizing effects of a thermo-electric feedback make the critical parameters different from those in the RB instability. The nonuniformity of the electric gravity arising from the finite variation of permittivity also affects the critical parameters. The characteristic constants of the GL equation are comparable with those for the RB convection. The heat transfer in the DEP convection is weaker than in the RB convection as a consequence of the feedback that impedes the convection.
\end{abstract}

\section{INTRODUCTION}

The application of an electric field $\boldsymbol{E}$ on a dielectric fluid gives rise to the electrohydrodynamic force density $\mathbf{f}_{E H D}[1]$ :

$$
\mathbf{f}_{E H D}=\rho_{f} \boldsymbol{E}-\frac{1}{2} E^{2} \nabla \epsilon+\nabla\left[\frac{\rho}{2}\left(\frac{\partial \epsilon}{\partial \rho}\right)_{T} E^{2}\right],
$$

where $\rho_{f}$ is the free electric charge density, $E$ is the magnitude of $\boldsymbol{E}$ and $T$ is the temperature. The electric permittivity and mass density of the fluid are denoted by $\epsilon$ and $\rho$, respectively. The first term is the electrophoretic force arising from the Coulomb forces that the field exerts on free charges. It is often the dominant component of $\mathbf{f}_{E H D}$ under a static or low frequency electric field. When the frequency $f$ is high compared with the viscous time scale $\tau_{\nu}=d^{2} / \nu(d$ : the length scale of a flow, $\nu$ : the kinematic viscosity of the fluid), the fluid cannot respond to the rapid variation of $\boldsymbol{E}$ and the electrophoretic force has no influence on its motion, as long as the charge density $\rho_{f}$ does not vary over the period $f^{-1}$. Under such a high frequency electric field, the second term of Eq. (1) called the dielectrophoretic (DEP) force becomes dominant, as $E^{2}$ has a static component. The third term is an electrostrictive force which would not influence flows when the fluid is incompressible and has no mobile boundaries [2].

The DEP force can arise when the fluid is subjected to a temperature gradient. The electric permittivity $\epsilon$ is a decreasing function of the temperature in the most of dielectric fluids and can be modeled by a linear relationship:

$$
\epsilon(\theta)=\epsilon_{1}(1-e \theta)
$$

\footnotetext{
* Harunori.Yoshikawa@univ-lehavre.fr
}

where $\epsilon_{1}$ is the electric permittivity at a reference temperature $T_{1}$ and $\theta$ is the temperature deviation from the reference temperature: $\theta=T-T_{1}$. A temperature gradient then results in a DEP force directed from low to high temperature regions in the fluid. This thermo-electric force can generate a thermal convection and, hence, it can be used to enhance heat transfer in dielectric fluids even under microgravity conditions.

The electric gravity $\boldsymbol{g}_{e}$ is often employed in the analysis of the DEP thermal convection for its intuitive comprehension. With use of Eq. (2), the DEP force can be developed as $-E^{2} \nabla \epsilon / 2=\nabla\left(e \theta \epsilon_{1} E^{2} / 2\right)-\theta e \boldsymbol{\nabla}\left(\epsilon_{1} E^{2} / 2\right)$. The first term is a gradient force that can be lumped with the pressure term in the dynamical equations of fluid motion. The second term can be regarded as a thermal buoyancy force, $-\rho \alpha \theta \boldsymbol{g}_{e}$ ( $\alpha$ : the coefficient of thermal expansion), due to an effective gravity $\boldsymbol{g}_{e}$ :

$$
\boldsymbol{g}_{e}=\frac{e}{\alpha \rho} \nabla\left(\frac{\epsilon_{1} E^{2}}{2}\right)
$$

which represents the variation of the electric energy stored in the dielectric fluid per volume. This electric gravity varies in space and time in general. The electric Rayleigh number $L=\alpha \Delta \theta g_{e 0} d^{3} / \kappa \nu$ is then introduced as control parameter of the DEP thermal convection, where $g_{e 0}$ is the characteristic value of the electric gravity $(d$, $\Delta \theta$ : the gap and the temperature difference between the electrodes, $\kappa$ : the thermal diffusivity).

Roberts [3], Turnbull [4] and Stiles et al. [5] have investigated the linear stability of a horizontal fluid layer between two parallel plane electrodes kept at different temperatures. Under the assumption that the DEP force was the primary component of the electrohydrodynamic force, they found its destabilizing effects on the stationary conductive state. Convection developed beyond critical values $L_{c}$ of the electric Rayleigh number, even in ordinarily stable thermal stratification where the temper- 
ature gradient was directed upwards. Under microgravity conditions, it was found that $L_{c}=2128.7$ with the critical wavenumber $k_{c}=3.226 / d$. Takashima \& Hamabata [6] have considered the same problem but in a vertical configuration of electrodes, assuming a vertical basic flow in the conductive regime. Instability occurred in different modes: hydrodynamic, thermal and electric, depending on the values of $L$ and the Grashof number $G r$. In the limit of small $G r$, the electric mode instability appeared at the same $L_{c}$ and $k_{c}$ as those in microgravity. The DEP convection in its nonlinear regime has not been investigated so much. Stiles et al. [7] have performed a weakly nonlinear analysis. They have estimated the heat transfer enhancement by convection for a Prandtl number $\operatorname{Pr}(=\nu / \kappa)=10$ and found $N u-1 \approx 0.8\left(L / L_{c}-1\right)$ in the vicinity of the criticality.

The similarity of the DEP thermal convection with the ordinary thermal convection has been noticed for a long time. However, the above critical parameters and Nusselt numbe are different from those in the RB convection. These differences have never been explained to the authors' knowledge. Furthermore, the DEP convection involves a parameter $\gamma_{e}=e \Delta \theta$ which has no corresponding part in the RB convection. This dimensionless permittivity variation $\gamma_{e}$ has been assumed to be small: $\left|\gamma_{e}\right| \ll 1$ in the previous theoretical works $[3,5-7]$, although it can be significant in some dielectric fluids with a large thermal coefficient e, e.g., in acetonitrile and nitrobenzene, $e \approx 0.2$.

In the present work, we examine the similarity of the DEP thermal convection in plane geometry with the RB convection by detailed theoretical considerations. We perform a linear stability analysis in a system with an infinite lateral extension with taking into account finite values of $\gamma_{e}$. A two-dimensional direct numerical simulation for a system with a large aspect ratio is also carried out for different $\operatorname{Pr}(\geq 1)$ to investigate the nonlinear behavior of the convection. Obtained results are analyzed from an energetic viewpoint and in the framework of the Ginzburg-Landau equation.

The governing equations of the DEP thermal convection are given in Sec. II with the basic conductive state. An energy equation is also given there for later discussions. The linear stability theory and its results are presented in Sec. III. The results of the direct numerical simulation are given in Sec. IV with their analysis by the Ginzburg-Landau (GL) equation. The heat transfer enhancement is also discussed. The final section is concerned with the conclusion.

\section{PROBLEM FORMULATION}

\section{A. Governing equations}

A dielectric fluid layer subjected to an alternating electric voltage $\sqrt{2} V_{0} \sin (2 \pi f t)$ and to a temperature differ-

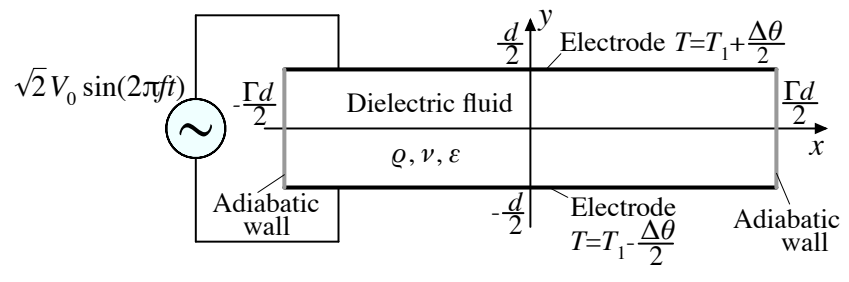

FIG. 1. Geometrical configuration of the problem.

ence $\Delta \theta$ is considered (Fig. 1). For an electric field with a high frequency compared to the viscous time scale $\tau_{\nu}$, only the time-averaged component of the DEP force can induce the convective motion of the fluid $[8,9]$. In this high frequency approximation, the equations of continuity, of motion and of heat conduction and the Gauss's law of electricity read in the electrohydrodynamic Boussinesq approximation [8]:

$$
\begin{aligned}
& \boldsymbol{\nabla} \cdot \boldsymbol{u}=0 \\
& \partial_{t} \boldsymbol{u}+\boldsymbol{u} \cdot \boldsymbol{\nabla} \boldsymbol{u}=-\boldsymbol{\nabla} \pi+\nabla^{2} \boldsymbol{u}-\frac{L}{P r} \theta \boldsymbol{g}_{e}, \\
& \partial_{t} \theta+\boldsymbol{u} \cdot \boldsymbol{\nabla} \theta=\frac{1}{P r} \nabla^{2} \theta, \\
& \boldsymbol{\nabla} \cdot[\epsilon(\theta) \boldsymbol{\nabla} \phi]=0 \quad \text { with } \boldsymbol{E}=-\boldsymbol{\nabla} \phi,
\end{aligned}
$$

where $\boldsymbol{u}$ is the two-dimensional velocity field: $\boldsymbol{u}=$ $u \hat{\boldsymbol{x}}+v \hat{\boldsymbol{y}}(\hat{\boldsymbol{x}}, \hat{\boldsymbol{y}}$ : the unit vectors along the $x$ - and $y$-axes), $\pi$ is the generalized pressure including electrohydrodynamic components and $\phi$ is the electric potential. The equations have been nondimensionalized with scales $d$ of length, $d^{2} / \nu$ of time, $V_{0}$ of electric potential and $\Delta \theta$ of temperature. In the present study the scale of time $d^{2} / \nu$ is more appropriate than the time scale of thermal diffusion $d^{2} / \kappa$, as the former is the smallest when $\operatorname{Pr}>1$, the case in which we are interested. The permittivity is scaled by $\epsilon_{1}$ and its thermal variation is given by $\epsilon=1-\gamma_{e} \theta$ according to Eq. (2).

These equations are completed by the boundary conditions on the electrodes at $y= \pm 1 / 2$ and on the walls at $x= \pm \Gamma / 2$ ( $\Gamma$ : the aspect ratio):

$$
\begin{array}{lr}
\boldsymbol{u}=0, \theta=\frac{1}{2}, \phi=1 & \text { at } y=-\frac{1}{2} \\
\boldsymbol{u} & =0, \theta=-\frac{1}{2}, \phi=0 \quad \text { at } y=\frac{1}{2} \\
\boldsymbol{u} & =0, \partial_{x} \theta=0, \partial_{x} \phi=0 \text { at } x= \pm \frac{\Gamma}{2}
\end{array}
$$

We have assumed that the walls are thermally adiabatic and made of a material with a small electric permittivity compared to the fluid (Eq. 10), considering the problem in its simplest configuration.

In the theoretical model formulated by Eqs. (4)-(10), we have considered an initially electroneutral fluid layer and have assumed that no space charge accumulation is induced by the electric field: $\rho_{f}=0$ in the bulk of the fluid during the entire development of convection 
flow. This assumption is valid under the following conditions: i) $f \gg \tau_{e}^{-1}, \tau_{m}^{-1}, \tau_{d}^{-1}$ and ii) $d \gg \lambda_{D}$, where $\tau_{e}$, $\tau_{m}$ and $\tau_{d}$ are the time scales of the charge relaxation, migration and diffusion processes, respectively. The Debye length $\lambda_{D}$ represents the thickness of the diffusion layer, which is an electrically charged layer constituting the outer part of the electric double layer formed on each fluid-electrode interface. Under condition i), the electric field varies too rapidly to alter the spatial distribution of charges in the fluid; under condition ii), the charge transport by the convection flows from the diffusion layer into the bulk will be negligible.

For a bipolar system consisting of positive and negative charges of the same magnitude $q$, the time scales and the Debye length are given by [10]:

$$
\begin{gathered}
\tau_{e}=\frac{\epsilon}{\sigma}, \quad \tau_{m}=\frac{h}{E\left(b_{+}+b_{-}\right)}, \quad \tau_{d}=\frac{h^{2}}{\frac{K_{+} b_{-}+K_{-} b_{+}}{b_{+}+b_{-}}}, \\
\lambda_{D}=\sqrt{\frac{\epsilon k_{B} T}{2 q^{2} n_{\infty}}}
\end{gathered}
$$

where $\sigma$ is the electric conductivity, $h$ is a length scale, $k_{B}$ is the Boltzmann constant, $n_{\infty}$ is the number density of both charges far from electrodes, and $b_{ \pm}$and $K_{ \pm}$are the mobilities and diffusion coefficients of positive/negative charges, respectively. In silicone oils, the time scales are estimated for the diffusion layer (i.e., $\left.h=\lambda_{D}\right)$ as $\tau_{e} \sim 10-10^{2} \mathrm{~s}, \tau_{d} \sim 10^{2} \mathrm{~s}$ and $\tau_{m} \sim$ $8.2 \times 10^{6} \nu^{1 / 2} E^{-1}\left(q / q_{e}\right)^{-1 / 2}[\mathrm{~s}]$ with $\lambda_{D} \sim 10^{-4} \mathrm{~m}$, where $q_{e}$ is the elementary charge and $\nu$ and $E$ are their values in the units of $\mathrm{m}^{2} / \mathrm{s}$ and $\mathrm{V} / \mathrm{m}$, respectively [11]. The migration time $\tau_{m}$ gives the smallest value among the three time scales at practical field strengths $\left(E \sim 10^{5}\right.$ $10^{6} \mathrm{~V} / \mathrm{m}$ ), being, e.g., $\tau_{m}=0.026 \mathrm{~s}$ for a silicone oil with $\nu=10^{-5} \mathrm{~m}^{2} / \mathrm{s}$. According to conditions i) and ii), the theoretical model (4)-(10) would enable to describe the flow in a layer of the latter oil, thicker than a few millimeters and subjected to an electric field with a frequency higher than $\tau_{m}^{-1}=38 \mathrm{~Hz}$.

\section{B. Conductive state}

When the imposed temperature difference is small, the purely conductive state $(\boldsymbol{u}=0)$ is established. The temperature and electric fields, $\theta=\bar{\theta}(y), \phi=\bar{\phi}(y)$ are then obtained analytically from Eqs. (6) and (7) with the boundary conditions (8)-(10).

$$
\bar{\phi}=\frac{\log \left(\frac{1+\gamma_{e} y}{1+\gamma_{e} / 2}\right)}{\log \left(\frac{1-\gamma_{e} / 2}{1+\gamma_{e} / 2}\right)}, \quad \bar{\theta}=-y
$$

This conductive state is independent of the aspect ratio $\Gamma$ thanks to the idealized boundary condition (10). It will therefore be considered as the basic state both in the linear stability theory performed for $\Gamma \rightarrow \infty$ and in the
DNS for a large but finite $\Gamma$. The electric gravity (3) in the conductive state is given by:

$$
\overline{\boldsymbol{g}}_{e}=\bar{g}_{e} \hat{\boldsymbol{y}} \quad \text { with } \quad \bar{g}_{e}=-\frac{1}{\left(1+\gamma_{e} y\right)^{3}} .
$$

We have chosen for scaling $\overline{\boldsymbol{g}}_{e}$ the electric gravity at the middle of the gap: $g_{e 0}=e \epsilon_{1} V_{0}^{2} \gamma_{e}^{3} / \rho \alpha d^{3}[\log \{(1-$ $\left.\left.\left.\gamma_{e} / 2\right) /\left(1+\gamma_{e} / 2\right)\right\}\right]^{2}$. The electric Rayleigh number $L$ in Eq. (5) is based on this characteristic gravity:

$$
L=\frac{\epsilon_{1} V_{0}^{2} \gamma_{e}^{4}}{\rho \kappa \nu}\left[\log \left(\frac{1-\gamma_{e} / 2}{1+\gamma_{e} / 2}\right)\right]^{-2}
$$

which recovers the electric Rayleigh number introduced in the previous works $[3,5-7]$ in the limit of small $\gamma_{e}$.

\section{Energy equation}

An equation that governs the evolution of the flow kinetic energy can be derived from the Navier-Stokes equation (5). Taking the inner product of the equation with $\boldsymbol{u}$ and integrating over the whole fluid domain, we have

$$
\frac{d K}{d t}=W_{B G}+W_{P G}-D_{\mathrm{v}},
$$

where $K, W_{B G}, W_{P G}$ and $D_{\mathrm{v}}$ are the flow kinetic energy, the work done by the basic electric gravity $\overline{\boldsymbol{g}}_{e}$, the work done by the perturbation electric gravity $\boldsymbol{g}_{e}^{\prime}=\boldsymbol{g}_{e}-\overline{\boldsymbol{g}}_{e}$ and the viscous dissipation, respectively. They are computed by integrating over the fluid domain the corresponding quantities per volume:

$$
\begin{aligned}
& \mathcal{K}=\left|\boldsymbol{u}^{\prime}\right|^{2} / 2, \\
& w_{B G}=-P r^{-1} L \theta^{\prime} \boldsymbol{u}^{\prime} \cdot \bar{g}_{e}, \\
& w_{P G}=-P r^{-1} L\left(\bar{\theta} \boldsymbol{u}^{\prime} \cdot \boldsymbol{g}_{e}^{\prime}+\theta^{\prime} \boldsymbol{u}^{\prime} \cdot \boldsymbol{g}_{e}^{\prime}\right), \\
& d_{\mathrm{v}}=\nabla \boldsymbol{u}^{\prime}:\left(\nabla \boldsymbol{u}^{\prime}\right)^{T}
\end{aligned}
$$

where the primes indicate perturbation quantities.

The basic electric gravity $\overline{\boldsymbol{g}}_{e}$ can be regarded as the counterpart in the DEP thermal convection to the Earth's gravity in the ordinary thermal convection. The perturbation electric gravity $\boldsymbol{g}_{e}^{\prime}$ represents a thermoelectric feedback associated with the electric field perturbations that arise from the permittivity variation due to temperature disturbances (Eq. 7). The contribution of the work $W_{P G}$ to the kinetic energy evolution is hence distinctive of the DEP convection.

\section{LINEAR STABILITY THEORY}

Governing equations (4)-(7) are linearized about the basic state (12). Developing perturbations into normal 
modes $e^{s t+i k x}$ with the complex growth rate $s$ and the wavenumber $k$, we have

$$
\begin{aligned}
& 0=i k U+D V, \\
& s U=\left(D^{2}-k^{2}\right) U-i k \Pi-\frac{L}{P r} \bar{\theta} G_{e x}, \\
& s V=\left(D^{2}-k^{2}\right) V-D \Pi-\frac{L}{P r} \bar{g}_{e} \Theta-\frac{L}{P r} \bar{\theta} G_{e y}, \\
& s \Theta=V+\frac{1}{P r}\left(D^{2}-k^{2}\right) \Theta, \\
& 0=-\gamma_{e}\left[D \bar{\phi} D+D^{2} \bar{\phi}\right] \Theta \\
& \quad+\left[\left(1+\gamma_{e} y\right)\left(D^{2}-k^{2}\right)+\gamma_{e} D\right] \Phi,
\end{aligned}
$$

where $(U, V, \Pi, \Theta, \Phi)$ are the normal mode amplitudes of the perturbations $\left(u^{\prime}, v^{\prime}, \pi^{\prime}, \theta^{\prime}, \phi^{\prime}\right)$, respectively, and the operator $D=d / d y$. The normal mode amplitudes $\left(G_{e x}, G_{e y}\right)$ of the perturbation electric gravity $\boldsymbol{g}_{e}^{\prime}$ are given by

$$
\begin{aligned}
G_{e x} \hat{\boldsymbol{x}}+ & G_{e y} \hat{\boldsymbol{y}}=\frac{1}{\gamma_{e}^{3}}\left[\log \left(\frac{1-\gamma_{e} / 2}{1+\gamma_{e} / 2}\right)\right]^{2} \\
& \times\left[i k D \bar{\phi} D \Phi \hat{\boldsymbol{x}}+\left(D \bar{\phi} D^{2} \Phi+D^{2} \bar{\phi} D \Phi\right) \hat{\boldsymbol{y}}\right] .
\end{aligned}
$$

On the electrodes the perturbations satisfy the following boundary conditions corresponding to Eqs. (8) and (9):

$$
U=D U=V=\Theta=\Phi=0 \quad \text { at } y= \pm \frac{1}{2} .
$$

The condition on the adiabatic walls (10) has been removed, as we consider a system with an infinite lateral extension $(\Gamma \rightarrow \infty)$ in the present linear theory.

The set of equations (20)-(24) are discretized by a spectral collocation method. All the unknown functions are developed into Chebyshev series and the equations are considered only at the Chebyshev-Gauss-Lobatto collocation points. The highest order of considered Chebyshev polynomials is set at 60 to ensure the convergence. The discretized governing equations are coupled with the boundary conditions (26) to form a generalized eigenvalue problem. Its eigenvalues and eigenvectors are computed by employing the $Q Z$-decomposition.

Marginal stability curves are obtained by solving the eigenvalue problem for different values of $L$ and $k$ at given $\operatorname{Pr}$ and $\gamma_{e}$. The minimum of a marginal curve gives the critical parameters $\left(k_{c}, L_{c}\right)$, which are found to be independent of the Prandtl number. The corresponding critical mode is stationary as in the RB problem $[12,13]$. The critical parameters for small $\gamma_{e}(<0.1)$ are constant, recovering the results of the existing theories $[3,5,6]$. For large $\gamma_{e}(>0.1)$, the critical parameters depend on it (Fig. 2): significant decrease and increase are found in $L_{c}$ and $k_{c}$, respectively.

The basic electric gravity $\overline{\boldsymbol{g}}_{e}$ provides energy to perturbation flow, i.e., $W_{B G}>0$ (Fig. 3). The mechanism driving the convection is hence the thermal buoyancy associated with a temperature perturbation $\theta^{\prime}$ in the gravity $\overline{\boldsymbol{g}}_{e}$, similar to the RB instability. In contrast, the perturbation gravity $\boldsymbol{g}_{e}^{\prime}$ dissipates energy, $W_{P G}<0$ (Fig. 3).

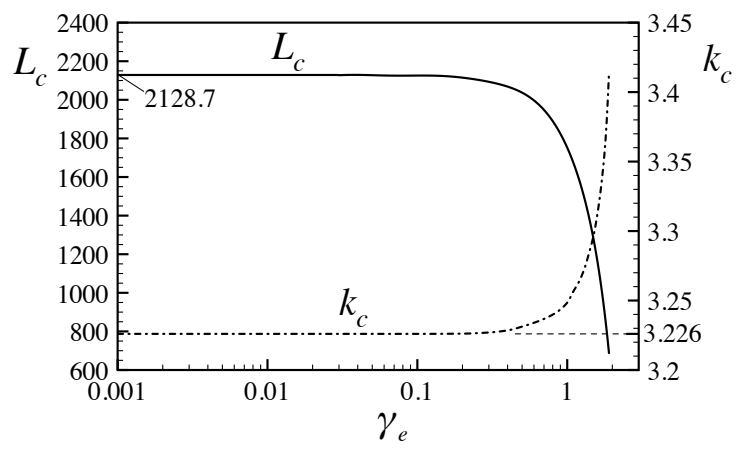

FIG. 2. Critical values of the electric Rayleigh number $L$ and the wavenumber $k$ as functions of the thermal variation of electric permittivity $\gamma_{e}$. The predictions of the previous linear stability theories $\left(L_{c}=2128.7, k_{c}=3.226\right)[3,5,6]$ are indicated for comparison.

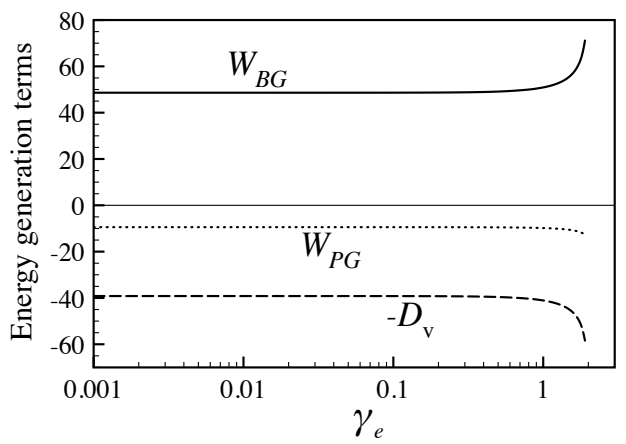

FIG. 3. Different terms in the energy equation (15) at critical conditions. The work done by the basic electric gravity $W_{B G}$, the work done by the perturbation electric gravity $W_{P G}$ and the viscous dissipation $D_{\mathrm{v}}$ are all normalized by twice the kinetic energy $K$.

This stabilization by $\boldsymbol{g}_{e}^{\prime}$ is absent in the RB convection and explains why $L_{c}$ at small $\gamma_{e}$ is larger than the critical Rayleigh number $R_{c}(=1708)$ in the RB convection in spite of the apparent similarity in the driving mechanism. The effect of $W_{P G}$ is also found to be more significant at small wavenumber, giving an explanation to $k_{c}=3.226$ that is larger than in the RB instability $\left(k_{c}=3.117\right)$.

For large $\gamma_{e}$, the work $W_{B G}$ is enhanced significantly, although the work $W_{P G}$ is almost constant (Fig. 3). This suggests that the basic electric gravity performs work more efficiently at large $\gamma_{e}$ than at small $\gamma_{e}$ to destabilize the conductive state at small $L_{c}$. Indeed, the spatial nonuniformity of the basic electric gravity $\overline{\boldsymbol{g}}_{e}$ is reinforced at large $\gamma_{e}$ (Eq. 13). Strong electric gravity field in the vicinity of the hot electrode $\left(y=-\frac{1}{2}\right)$ provides energy to the fluid locally (Fig. 4a) and provokes the instability within a fluid sublayer attached to the electrode. As a consequence, the kinetic energy of developed flow is also concentrated in the region near the hot electrode (Fig. 4b): the convection develops within an effective 

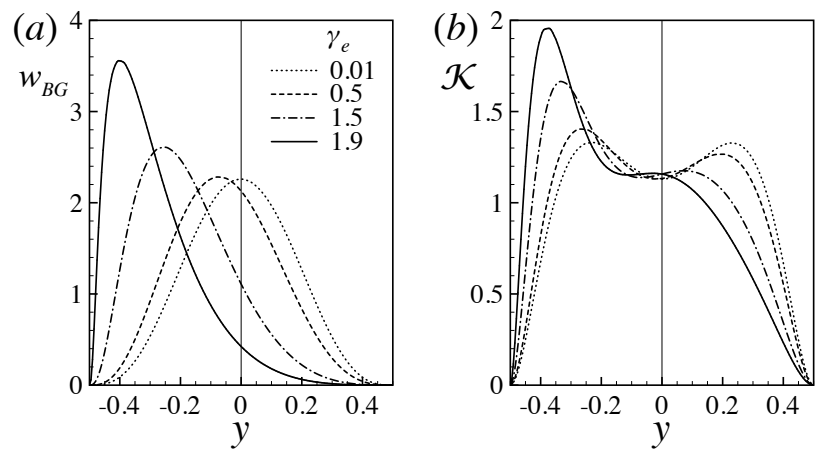

FIG. 4. Profiles of the local work done by the basic electric gravity $w_{B G}$ and the local flow kinetic energy $\mathcal{K}$, averaged along the $x$-direction and normalized, respectively, by $W_{B G}$ and $K$.

sublayer of a small thickness. This explains the observed large critical wavenumbers (i.e., small wavelengths) at large $\gamma_{e}$.

\section{DIRECT NUMERICAL SIMULATION}

We have considered the nonlinear behavior of the DEP thermal convection for a small $\gamma_{e}(=0.03)$ and a large aspect ratio $\Gamma=114$, solving the set of partial differential equations (4) $-(7)$ with the boundary conditions (8)(10) by the finite element method implemented in a commercial software (COMSOL Multiphysics ${ }^{\mathrm{TM}}$ ). Numerical grids are made of identical rectangles with sides of $\Delta x=0.15$ and $\Delta y=0.1$ so that the fluid domain is divided by 760 and 10 along the $x$ - and $y$-directions, respectively. The backward differentiation formula is used for the time integration. The convergence of computation was verified by grid refinements. The initial fields are specified as null for the velocities, the temperature and the electric field: the solved problem corresponds to a situation where the electric potential $V_{0}$ and the temperature difference $\Delta \theta$ are imposed instantaneously on a steady isothermal fluid layer at $t=0$.

For a value of $L$ larger than $L_{c}$, small disturbances grow exponentially to develop convection cells. This linear growth stage is followed by a saturation where hot and cold cells are shifted toward the low and high temperature electrodes, respectively, yielding a net heat transfer enhancement by the convection (Fig. 5a, b). The cells have a well-defined wavenumber $k$ along the $x$-direction. Its Fourier spectrum has a sharp peak with a width around $\Delta k=0.05$ arising from the perturbation suppression at the lateral walls.

\section{A. Description by the Ginzburg-Landau equation}

The bifurcation at $L=L_{c}$ is found to be supercritical. As the critical mode is stationary and has a finite
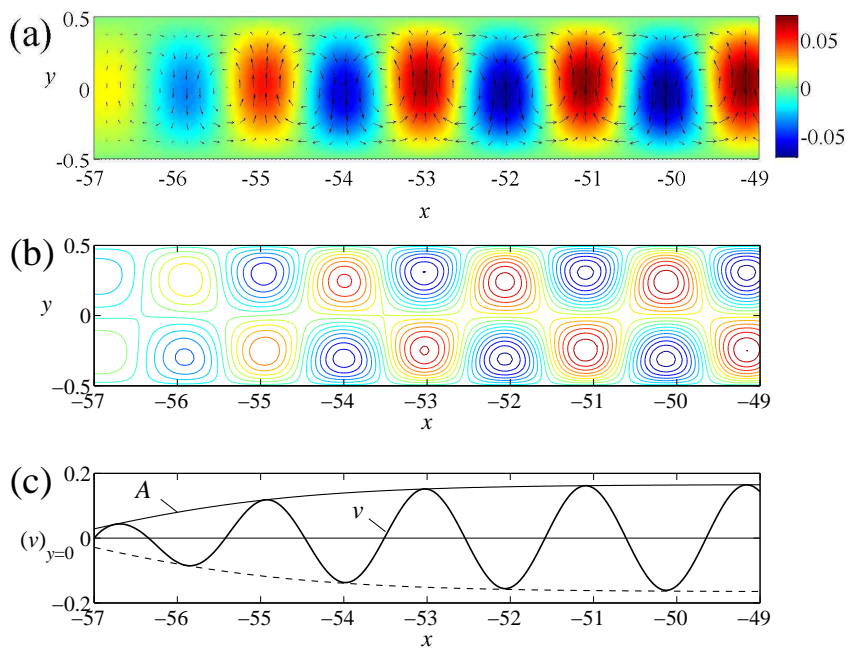

FIG. 5. Convection flow in the saturated state for $\operatorname{Pr}=10$ at $L=2200$ : (a) velocity (arrows) and perturbation temperature (color), (b) equipotentials of perturbation electric field and (c) the profile of the transversal velocity component $v$ at the middle of the gap $(y=0)$. The left end $(x=-57)$ corresponds to one of the adiabatic lateral walls.
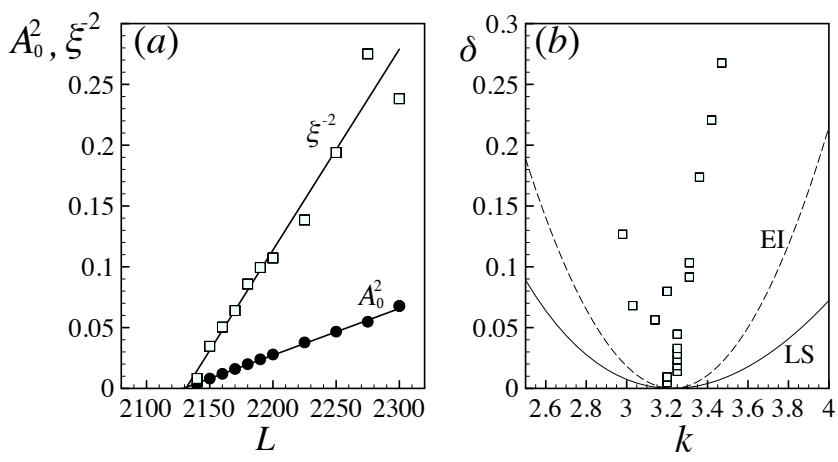

FIG. 6. Behavior of (a) the healing length $\xi$ and the saturation amplitude $A_{0}$ and (b) the wavenumber $k$ in the vicinity of the criticality $(\operatorname{Pr}=10)$. The supercriticality $\delta\left(=L / L_{c}-1\right)$ is computed with $L_{c}=2130$. In (b) also shown are the marginal curve obtained in the linear stability theory (LS) and the Eckhaus instability boundary (EI): $\delta=3 \xi_{0}^{2}\left(k-k_{c}\right)^{2}$.

wavenumber $k_{c}$ (i.e., Type I-s instability [14]), the behavior of the perturbation amplitude in the weakly nonlinear regime is hence expected to be described by the Ginzburg-Landau (GL) equation:

$$
\tau_{0} \partial_{t} A=\delta A+\xi_{0}^{2} \partial_{x}^{2} A-\ell|A|^{2} A
$$

where $\delta$ is the supercriticality $\delta=L / L_{c}-1$. The constants $\tau_{0}, \xi_{0}$ and $\ell$ are characteristics of a given system. In the present work, the envelop of the velocity profile $v=v(x)$ at the middle of the gap $(y=0)$ will be taken as the amplitude $A$.

In the saturated state, the amplitude is constant far 
TABLE I. Critical parameters and characteristic constants of the Ginzburg-Landau equation (27) determined from the DNS $\left(\gamma_{e}=0.03\right)$.

\begin{tabular}{c|cccc}
$P r$ & $L_{c}$ & $\tau_{0}$ & $\xi_{0}$ & $\ell$ \\
\hline 1 & 2128.2 & 0.0783 & 0.388 & 0.0121 \\
10 & 2128.9 & 0.590 & 0.377 & 1.15 \\
100 & 2130.1 & 5.62 & 0.380 & 118 \\
1000 & 2130.0 & 55.9 & 0.381 & 11800
\end{tabular}

from the adiabatic walls (Fig. 5c) where the GL equation predicts $|A|=\sqrt{\delta / \ell}=\sqrt{\left(L / L_{c}-1\right) / \ell}\left(=A_{0}\right)$. The critical electric Rayleigh number $L_{c}$ can be deduced from the intersection point of the $A_{0}^{2}$-line with the $L$-axis (Fig. 6a). We find $L_{c}=2130$, independently from the Prandtl number (Table I) as in the linear stability theories $[3,4,6,7]$. The perturbation suppression by the lateral walls affects little the critical parameters, since the aspect ratio $\Gamma$ is large.

In a large system, the solution of the GL equation in the vicinity of a suppressing lateral boundary is given by $A=e^{i \Phi} \sqrt{\delta / \ell} \tanh \left[\left(x-x_{w}\right) / \xi\right]$. On the first order, $x_{w}$ is identical to the wall position and the phase $\Phi$ is an arbitrary constant. The constant $\xi$ represents a distance over which perturbations heal from the suppression at the boundary and is given by $\xi=\sqrt{2} \xi_{0} \delta^{-1 / 2}$. The hyperbolic tangent profile gives correct envelops to the variation of $(v)_{y=0}$ (Fig. 5c). The determined healing length $\xi$ behaves as the theoretical predictions: $\xi^{-2}$ increases linearly with $L$ and intersects the $L$-axis at $L=L_{c}$ (Fig. 6a).

The values of the characteristic constants $\tau_{0}, \xi_{0}$ and $\ell$ were found from the linear fits for $\delta / \tau_{0}$ (the growth rate), $\xi^{-2}$ and $A_{0}^{2}$ as functions of the supercriticality $\delta$. Table I shows the determined values of the constants for different values of $\mathrm{Pr}$. The characteristic time $\tau_{0}$ increases linearly with $P r$ and can be correlated by the same relationship derived for the RB convection: $\tau_{0}=(\operatorname{Pr}+0.5117) / 19.65[15]$. The values of $\xi_{0}$ do not vary with $\mathrm{Pr}$ and are also identical to the value found in the RB problem: $\xi_{0}=0.385$. The determined $\xi_{0}$ enables to draw the stability boundary of the Eckhaus instability: $\delta=3 \xi_{0}^{2}\left(k-k_{c}\right)^{2}$ (Fig. 6b). The wavenumbers obtained in the DNS are inside the stable zone. The constant $\ell$ behaves as $\ell \propto P r^{2}$, implying $|A| \propto \operatorname{Pr}^{-1}$. Since we have chosen the envelop of the velocity $v$ as the amplitude $A$, this means that the convection velocity is scaled by $\operatorname{Pr}^{-1} \cdot(\nu / d)=\kappa / d$, as in the ordinary thermal convections.

\section{B. Heat transfer enhancement}

The heat transfer is enhanced by the developed convection for $L>L_{c}$. Figure 7 shows the Nusselt number

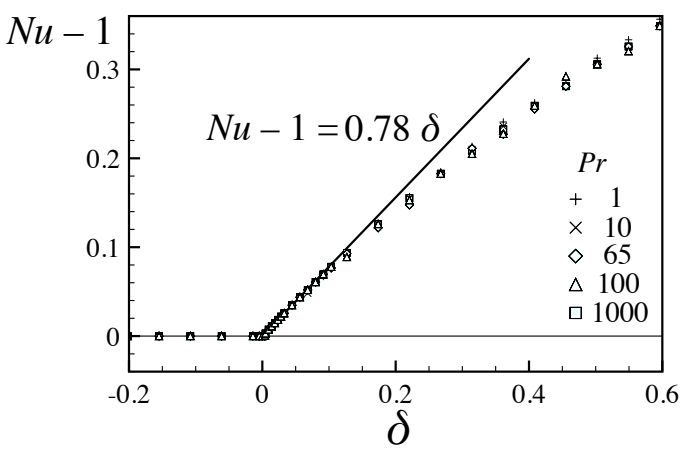

FIG. 7. Heat transfer enhancement by saturated convection flows. The Nusselt number $N u$ is shown as a function of the supercriticality $\delta$ for different Prandtl numbers $\operatorname{Pr}\left(\gamma_{e}=\right.$ $0.03)$.

$N u$ as a function of the supercriticality $\delta$ :

$$
N u=\frac{1}{\Gamma}\left(-\int_{-\Gamma / 2}^{\Gamma / 2} \partial_{y} \theta d x+\operatorname{Pr} \int_{-\Gamma / 2}^{\Gamma / 2} v \theta d x\right) .
$$

This number is the ratio of the total heat transfer to the conductive heat transfer in the basic state (12). The behavior of $N u$ is correlated by $N u-1=0.78 \delta[16]$ for small spercriticality $\delta$, indifferently from the Prandtl number $\operatorname{Pr}(\geq 1)$. The coefficient 0.78 agrees with its value obtained in a weakly nonlinear analysis for $\mathrm{Pr}=$ $10[7]$.

In the $\mathrm{RB}$ convection, the relationship $N u-1 \approx$ $1.43\left(R / R_{c}-1\right)[17]$ has been found for steady roll modes insensitively from $\operatorname{Pr}(\gtrsim 1)$. The coefficient of the supercriticality found for the DEP convection is hence substantially smaller than in the RB convection. This difference is too large to be explained by the spatial nonuniformity of the basic gravity (13) or by the presence of the lateral walls: the former effect will be of the order of $O\left(\gamma_{e}\right)=10^{-2}$ and the latter one will be about $O\left(\Gamma^{-1}\right)=10^{-2}$. In the linear stability theory, it was found that $\boldsymbol{g}_{e}^{\prime}$ tends to dissipate the kinetic energy of flow (Fig. 3). This tendency persists even after the saturation (Fig. 8). The reduction in convective heat transfer by the perturbation electric gravity will be of the order of $W_{P G} / W_{B G} \approx 0.3$, agreeing with the relative difference between the coefficients in the $N u$ correlations. The impeding effect of the perturbation electric gravity $\boldsymbol{g}_{e}^{\prime}$ on convection flows hence gives an explanation to the weaker heat transfer enhancement in the DEP convection than in the RB convection.

\section{CONCLUSIONS}

In the present study, the similarity of the DEP thermal convection in plane geometry with the RB convection has been examined in detail by a linear stability 


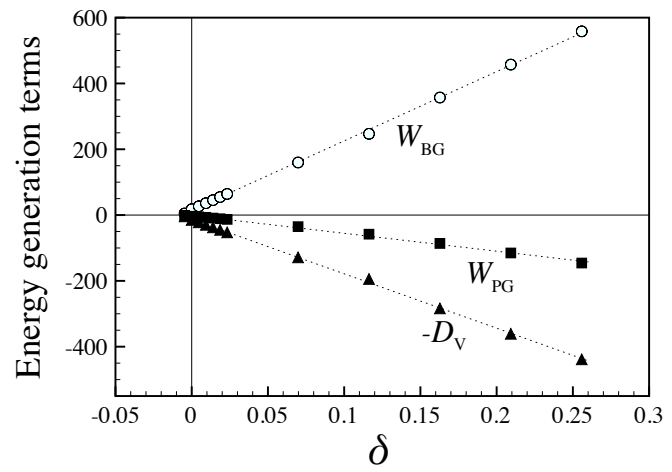

FIG. 8. Different terms in the energy equation (15) in the vicinity of the criticality in saturated states $(\operatorname{Pr}=10)$. The work done by the basic electric gravity $W_{B G}$, the work done by the perturbation electric gravity $W_{P G}$ and the viscous dissipation $D_{\mathrm{v}}$ are all normalized by twice the kinetic energy $K$.

analysis and a direct numerical simulation. The problem was formulated with regarding the DEP force as thermal buoyancy due to the electric gravity (3). The difference from the RB convection was highlighted by introducing the perturbation electric gravity $\boldsymbol{g}_{e}^{\prime}$, which represents the thermo-electric feedback, and by considering finite values of the permittivity variation $\gamma_{e}$, which is associated with the nonuniformity in the basic gravity $\overline{\boldsymbol{g}}_{e}$ (Eq. 13).

The linear stability theory revealed that the electric gravity perturbation dissipates flow kinetic energy and tends to stabilize the basic conductive state. The critical parameters $L_{c}$ and $k_{c}$ are hence different from those at the RB instability even when $\overline{\boldsymbol{g}}_{e}$ is almost uniform over the gap. When the nonuniformity of $\overline{\boldsymbol{g}}_{e}$ is important $\left(\gamma_{e}>0.1\right)$, the instability occurs within a fluid sublayer attached on the hot electrode where the electric gravity is strong and provides energy efficiently to convection flows. As a consequence, the critical values of $L$ and $k$ decreases and increases, respectively, from their values at small $\gamma_{e}$.

The results obtained by the DNS showed that the convection develops when $L>L_{c}$ with wavenumbers inside the Eckhaus stable zone. The convection flow in the nonlinear regime is well described by the GL equation. The determined characteristic time $\tau_{0}$ increases with $\operatorname{Pr}$ in the same manner as that obtained for the RB convection. The characteristic length $\xi_{0}$ is constant, being also the same as that in the RB convection. In spite of this similarity, impeding effects of the perturbation electric gravity persists in the nonlinear regime and results in a heat transfer enhancement weaker than in the RB convection.

\section{ACKNOWLEDGMENTS}

This work has benefited from a partial financial support from the CNES (Centre National des Etudes Spatiales). MTF had been supported by the doctor scholarship from the French Ministry of Higher Education and Research. HNY wishes to thank the Universite du Havre and the FEDER (Fonds Européen de Développement Régional) for his postdoctoral grant. The authors have had enriched discussions during the GEOFLOW Topical Team Meetings in Cottbus.
[1] L. D. Landau and E. M. Lifshitz, Electrodynamics of Continuous Media, 2nd ed., Landau and Lifshitz Course of Theoretical Physics, Vol. 8 (Elsevier ButterworthHeinemann, Burlington, Massachusetts, 1984).

[2] T. B. Jones, Advances in Heat Transfer 14, 107 (1979).

[3] P. H. Roberts, Q. J. Mech. Appl. Math. 22, 211 (1969).

[4] R. J. Turnbull, Phys. Fluids 12, 1809 (1969).

[5] P. J. Stiles, Chem. Phys. Lett. 179, 311 (1991).

[6] M. Takashima and H. Hamabata, J. Phys. Soc. Jpn 53, 1728 (1984).

[7] P. J. Stiles, F. Lin, and P. J. Blennerhassett, Phys. Fluids A 5, 3273 (1993).

[8] R. J. Turnbull and J. R. Melcher, Phys. Fluids 12, 1160 (1969).

[9] I. M. Yavorskaya, N. I. Fomina, and Y. N. Belyaev, Acta Astronaut. 11, 179 (1984).

[10] J. R. Melcher, Continuum Electromechanics (The MIT Press, 1981).

[11] In Eqs. (11), the mobilities $b_{ \pm}$can be computed as $b_{ \pm}=a_{ \pm} / \rho \nu$ for highly insulating liquids with $a_{+}=$ $1.5 \times 10^{-11} \mathrm{C} / \mathrm{m}$ and $a_{-}=3.0 \times 10^{-11} \mathrm{C} / \mathrm{m}$. The diffusion coefficients $K_{ \pm}$are given by the Einstein relation
$K_{ \pm}=k_{B} T b_{ \pm} / q[10]$.

[12] S. Chandrasekhar, Hydrodynamic and Hydromagnetic Stability (Dover, New York, 1961).

[13] H. N. Yoshikawa, O. Crumeyrolle, and I. Mutabazi, Phys. Fluids 25, 024106 (2012).

[14] M. C. Cross and P. C. Hohenberg, Rev. Mod. Phys. 65, 851 (1993).

[15] M. C. Cross, Phys. Fluids 23, 1727 (1980), The equation for $\tau_{0}$ given in the present paper is different by a factor $\mathrm{Pr}^{-1}$ from the equation given in this reference due to the difference in the scale of time in nondimensionalization.

[16] This correlation enables us to compare the values of the constant $\ell$ for the DEP and RB convections. Cross [15] has found $\ell \approx 0.70$ for the $\mathrm{RB}$ convection with scaling the amplitude $A$ by the relationship $(N u-1) R / R_{c}=|A|^{2}$ where $R$ is the Rayleigh number and $R_{c}$ is its critical value. Applying the same scaling to the $\mathrm{DEP}$ convection, we have $\ell=1 / 0.78=1.3$.

[17] A. Schlüter, D. Lortz, and F. Busse, J. Fluid Mech. 23, 129 (1965). 